\title{
Nonlinear Modeling and Identification of an Autonomous Tractor-Trailer System
}

\author{
Erkan Kayacan $^{\mathrm{a}}$, Erdal Kayacan ${ }^{\mathrm{b}}$, Herman Ramon $^{\mathrm{a}}$, Wouter Saeys ${ }^{\mathrm{a}}$ \\ ${ }^{a}$ Department of Biosystems (BIOSYST), Division of Mechatronics, Biostatistics and Sensors (MeBioS), University of Leuven (KU Leuven), \\ Kasteelpark Arenberg 30, 3001, Leuven, Belgium. Tel. +32 16 377089, Fax: +32 16321994 \\ e-mail: \{erkan.kayacan, herman.ramon,wouter.saeys\}@biw.kuleuven.be \\ ${ }^{b}$ School of Mechanical \& Aerospace Engineering, Nanyang Technological University, 50 Nanyang Avenue, Singapore 639798 \\ e-mail: erdal@ntu.edu.sg
}

\begin{abstract}
This paper presents the nonlinear modeling of the yaw and longitudinal dynamics of a tractor-trailer system. First, the yaw dynamic models of both the tractor and trailer are derived considering the lateral forces and side-slip angles. In order to be able to calculate the side-slips precisely, the relaxation length approach is preferred. Since the obtained yaw dynamic models are nonlinear, a constrained nonlinear optimization problem is formulated for the parameter estimation. Second, the longitudinal dynamic model for the system is derived based-on the static and dynamic responses. The static model consist of two inputs, the hydrostat position and the diesel engine speed, and one output, the longitudinal speed of the system. Afterwards, a dynamic model is proposed to define the dynamic effect between the output of the static model and the actual longitudinal speed. Third, the mathematical models of the steering mechanisms both for the tractor and trailer are identified. Consequently, a complete nonlinear dynamic model for the tractor-trailer system is obtained. The overall resulting model is thought to provide useful physical insight on such a complex mechatronic system, and can serve as the input for model based controller design.
\end{abstract}

Keywords: tractor-trailer system, agricultural vehicle, autonomous guidance, modeling, parameter estimation, system identification.

\section{Introduction}

Automation of agricultural production machines is thought to be up-and-coming for farmers as it can lighten the job of the operator, especially now that more and more actions in addition to driving the vehicle, are asked from him. For instance, the operation of the trailer during tillage and planting can be mentioned as an additional action for an operator. Even in a single-task operation, in addition to time pressure, operators have to deal with challenging working conditions such as high temperature, dust, etc. in the field. In such cases, the accuracy and efficiency of the planting or harvesting decrease as he gets tired and loses his concentration over time. As a solution to the aforementioned problems, automatic guidance of agricultural production machines has been proposed benefitting from several advanced control algorithms to improve the efficiency and productivity of various field operations such as tillage, planting and harvesting.

Automatic guidance of agricultural production machines not only improves the accuracy of the field operations but also reduces the overlap resulting in less crop damage, compaction and rutting [14]. Without loss of generality, there exist two basic methods for the realization of off-road vehicle automatic guidance: local positioning systems (vision or laser-based sensors) and global positioning systems (GPSs). Whereas mostly the introductory applications in the 1970s relied on the former [13, 24], recent implementations in the 2000s have started using the latter method [3, 16] in which highly accurate GPSs are used inspired by several successful applications in the navigation of airplanes and marine vehicles. As a local positioning system, although the vision-based systems are relatively cheap to implement, they have major disadvantages in outdoor environments, e.g. to be very sensitive to light conditions [10]. Realtime kinematic (RTK) GPSs have several advantages over local positioning systems such as the availability of the absolute position of the vehicle instead of the local coordinates, world-wide availability, ease of use as well as some disadvantages such as its relatively high cost, and the sensitivity to the presence of trees and buildings. In this investigation, two GPS antennas are used to determine the global positions of both the tractor and trailer. 
There are two factors which determine the performance of a model-based controller: accomplishment of the model and well-tuning of the controller coefficients. So, a prerequisite for an accurate performance of such controllers is the achievement of a precise mathematical model of the system to be controlled. Several researchers have investigated the automatic guidance of the agricultural tractors by using model-based controllers [16, 1,2, 19]. The common point of the studies mentioned up to now is that all these controllers rely on the kinematic tractor and trailer models which do not include the dynamics of the system. From the implementation point of view, these models are easy and simple to be dealt with. However, since the kinematic models neglect the important dynamics, the performance of the designed guidance systems based-on such models is limited [4]. The reason for this poor performance is that the equipments to automate tractor-trailer systems are highly nonlinear; they include saturation and dead-band regions. In addition to their complex dynamics, these machines have to work under highly uncertain and variable soil conditions. In such cases, the model-based controllers, especially linear time invariant controllers, have to be tuned very conservatively. By this conservative tuning, robustness of the controller is obtained at the price of performance. On the other hand, to achieve an acceptable accuracy in a field operation, $1.5 \mathrm{~cm}$ guidance accuracy is needed [25]. The achievement of such a strict control specification under uncertain working environments with highly nonlinear vehicle and implement dynamics requires of a better understanding of the dynamic behaviour of the systems to be controlled [14]. To promote the design of better model-based controllers, a dynamic model of a tractor-trailer system has been elaborated in this study. Particular attention is given to the analysis of the dynamics at longitudinal speeds within the range of 0-2 $\mathrm{m} / \mathrm{s}$ where most of the field operations are realized except some special operations such a spraying.

In this study, a tricycle model, in which it is assumed that the lateral forces on the left and right wheels are equal and can be summed, is used to derive the equations of motion of the tractor-trailer system. The tricycle dynamic model in this investigation is similar to the ones in $[14,7]$. However, the main contribution of the proposed model in this study is that there are no small steering angle assumptions for the tractor, trailer and hitch point angle resulting in a fact that the overall system is capable of following curvilinear trajectories. These assumptions result in a nonlinear tricycle dynamic model for the tractor-trailer system.

The body of the paper contains six sections: In Section 2, the real-time system description is given. In Section 3 , the dynamic equations of the autonomous tractor-trailer system are presented. The longitudinal dynamics model is given in Section 4, and the steering mechanism dynamics models for the tractor and implement are presented in Section 5. Finally, some conclusions are drawn in Section 6.

\section{Experimental Set-up Description}

The global aim of the real-time experiments in this investigation is to be able to model and identify the small scale agricultural tractor-trailer system shown in Fig. 1. Two GPS antennas are located straight up the center of the tractor rear axle and the center of the trailer to provide highly accurate positional information. They are connected to a Septentrio AsteRx2eH RTK-DGPS receiver (Septentrio Satellite Navigation NV, Leuven, Belgium) with a specified position accuracy of $2 \mathrm{~cm}$ at a $5-\mathrm{Hz}$ sampling frequency. The Flepos network supplies the RTK correction signals via internet by using a Digi Connect WAN $3 G$ modem.

The GPS receiver and the internet modem are connected to a real time operating system (PXI platform, National Instruments Corporation, Austin, TX, USA) through an RS232 serial communication. The PXI system acquires the steering angles and the GPS data, and controls the tractor-trailer system by sending messages to actuators. A laptop connected to the PXI system by WiFi functions as the user interface of the autonomous tractor. The algorithms are implemented in LabVIEW ${ }^{T M}$ version 2011 (National Instruments, Austin, TX, USA). They are executed in real time on the PXI and updated at a rate of 5-Hz.

The angle of the front wheels of the tractor is measured using a potentiometer mounted on the front axle yielding an angle measurement resolution of $1^{\circ}$. The position of the electro-hydraulic valve on the trailer is measured by using an inductive sensor with $1^{\circ}$ precision. The longitudinal speed of the tractor is controlled by using an electro-mechanic valve. The wheel speed control system consists of a cascade of two PID controllers. The proportional-derivativeintegral (PID) controllers in outer closed-loop and inner closed-loop are generating the desired pedal position with respect to the speed of the tractor and the voltage for the spindle actuator (LINAK A/S, Denmark) for the pedal position, respectively. In Figure 2, the spindle actuator for the hydrostat position (Fig. 2(a)), the potentiometer for the steering angle of the tractor(Fig. 2(b)) and the electro-hydraulic valve for the trailer (Fig. 2(c)) respectively are shown. 


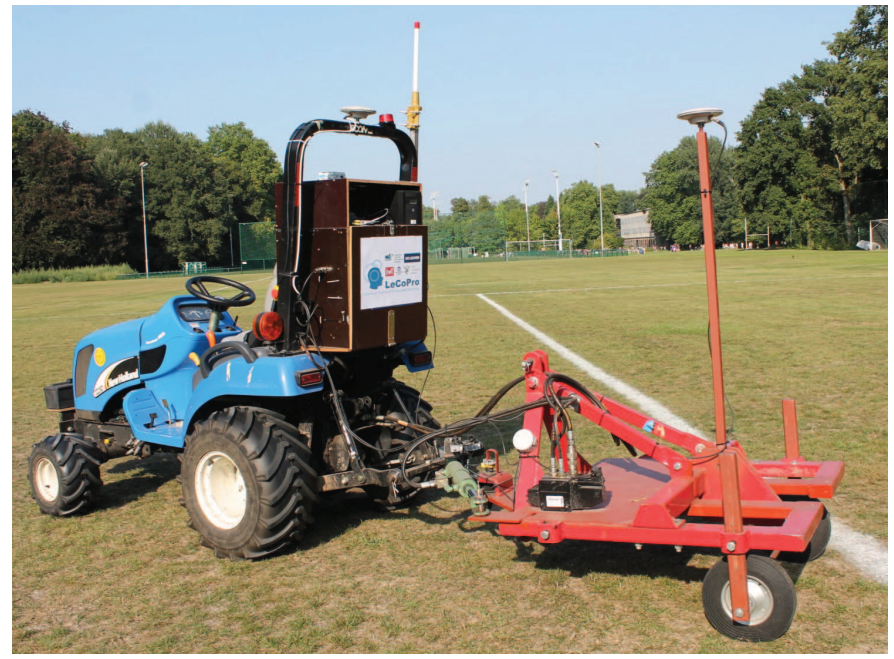

Figure 1: The tractor-trailer system

The rpm of the diesel engine has been measured by using a hall effect sensor (Hamlin, USA) which is connected to the shaft between the diesel engine and oil pump.

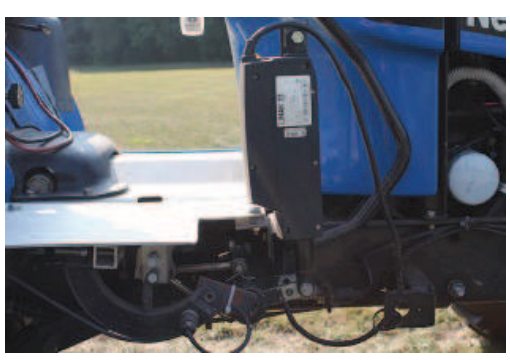

(a)

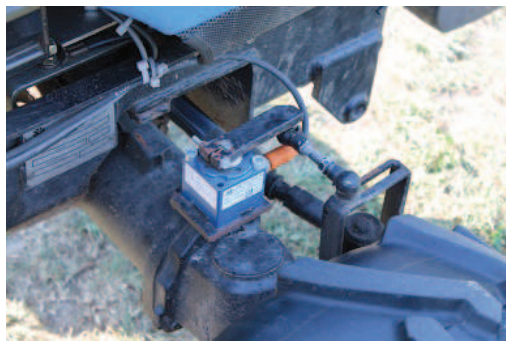

(b)

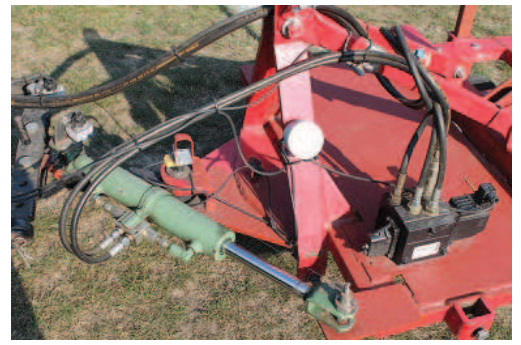

(c)

Figure 2: (a) Hydrostat spindle actuator (b) Steering angle potentiometer (c) Trailer actuator

\section{Modeling of the Tractor-Trailer Yaw Dynamics}

As the driving speeds of the tractor-trailer combination is rather limited, it is reasonable to assume that the lateral forces on the left and right wheels are equal and can be summed. Therefore, the tractor-trailer system is modeled in $2 \mathrm{D}$ as a tricycle system [14, 7], which is schematically illustrated in Fig. 3. However, in contrast to previous studies no assumptions are made with respect to the size of the steering angles in order to also accurately describe the system behavior on curvilinear trajectories. This results in a non-linear dynamic model of the tractor-trailer system.

The tractor and trailer rigid bodies are mechanically linked to each other by the drawbar. There are two revolute joints which connect the drawbar to the tractor and the drawbar to the trailer as illustrated in Fig. 3. The dynamics of the drawbar are negligible due to its low weight, such that it can be assumed that there is only one revolute joint in the formulation. Therefore, only one revolute joint is included in the rigid multibody model.

The velocities, side-slip angles and forces on the rigid body of an autonomous tractor-trailer system are schematically illustrated in Figs. 4-5. The connection point $H$ between the tractor and trailer in Figs. 4-5 refers to revolute joint $2\left(R J^{2}\right)$ in Fig. 3.

The notations used in the following (see also Figs. 4 and 5) are summarized in Table 1. 


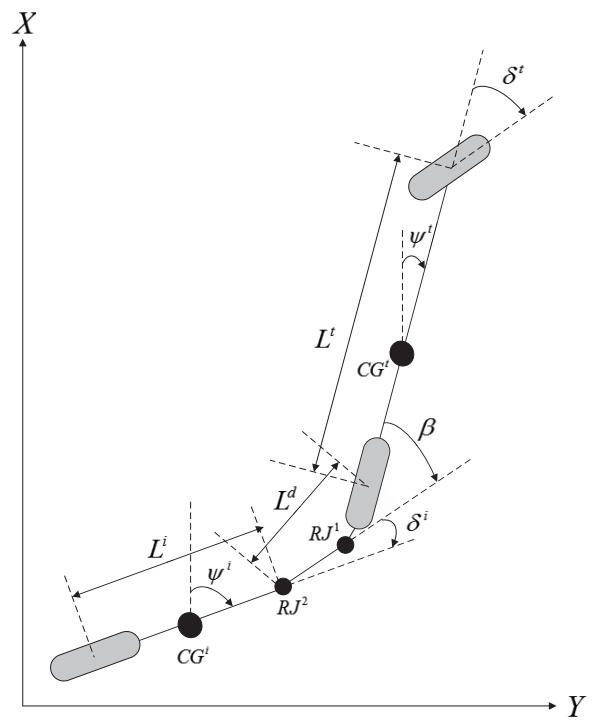

Figure 3: The schematic illustration of the tricycle model for an autonomous tractor-trailer system

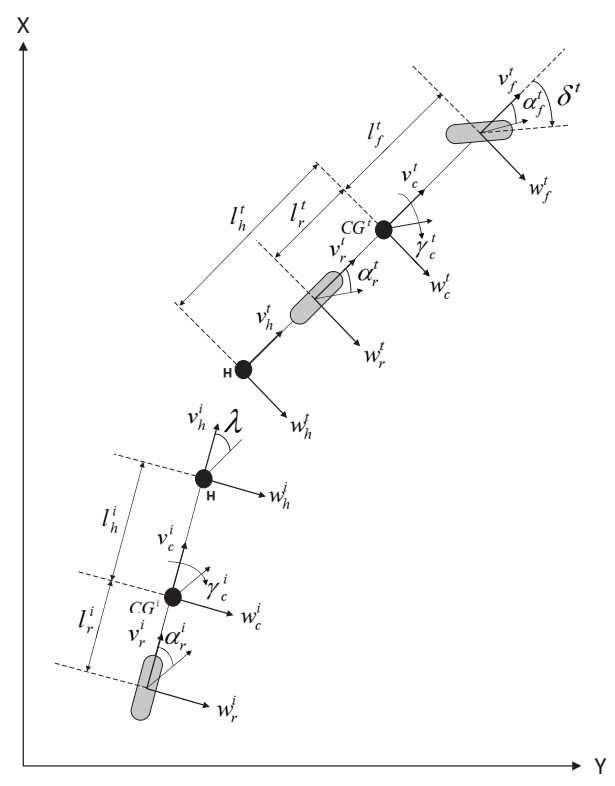

Figure 4: The dynamics tricycle model for a tractor-trailer system: velocities and slip angles on the rigid body of the system

\subsection{Vehicle Dynamics}

The yaw dynamic model is derived based-on the following assumptions:

- The traction forces are neglected,

- The aerodynamic forces are neglected,

- The tire moments are small such that these can be neglected, 


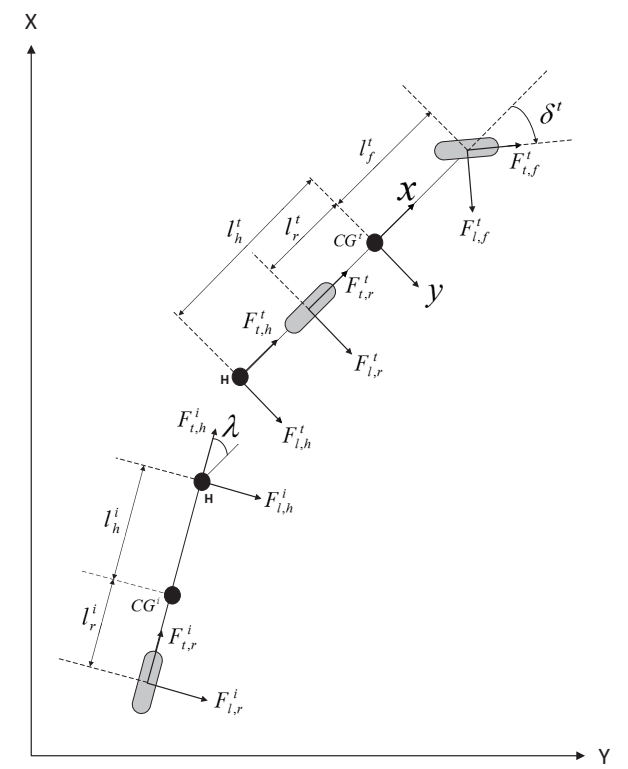

Figure 5: The dynamics tricycle model for a tractor-trailer system: forces on the rigid body of the system

- The pitch and roll dynamics are neglected.

The lateral motion dynamics of the tractor can be written based on Newton's second law as follows:

$$
m^{t}\left(\dot{w}_{c}^{t}+v_{c}^{t} \gamma_{c}^{t}\right)=F_{t, f}^{t} \sin \delta^{t}+F_{l, f}^{t} \cos \delta^{t}+F_{l, r}^{t}+F_{l, h}^{t}
$$

where $m^{t}, w_{c}^{t}, v_{c}^{t}, \gamma_{c}^{t}, \delta^{t}, F_{t, f}^{t}, F_{l, f}^{t}, F_{l, r}^{t}$ and $F_{l, h}^{t}$ represent the mass, the lateral and longitudinal velocities, the yaw rate of the center of gravity $(\mathrm{CG})(\mathrm{CG})$ of the tractor, the steering angle of the front wheel of the tractor, the traction and lateral forces on the front wheel of the tractor, the lateral forces on the rear wheel of the tractor and hitch point, respectively.

The yaw motion dynamics of the tractor can similarly be written as follows:

$$
I^{t} \dot{\gamma}_{c}^{t}=l_{f}^{t}\left(F_{t, f}^{t} \sin \delta^{t}+F_{l, f}^{t} \cos \delta^{t}\right)-l_{r}^{t} F_{l, r}^{t}-l_{h}^{t} F_{l, h}^{t}
$$

where $I^{t}, l_{f}^{t}, l_{r}^{t}$ and $l_{h}^{t}$ represent the moment of inertia of the tractor, the distance between the front axle and the CG of the tractor, the distance between the rear axle and the CG of the tractor, the distance between the hitch point and the $\mathrm{CG}$ of the tractor, respectively. The yaw inertial moment of the tractor can be estimated as proposed by [8]:

$$
I^{t}=m^{t} l_{f} l_{r}
$$

By neglecting the traction forces equations (1) and (2) can be re-written as follows:

$$
\begin{aligned}
m^{t}\left(\dot{w}_{c}^{t}+v_{c}^{t} \gamma_{c}^{t}\right) & =F_{l, f}^{t} \cos \delta^{t}+F_{l, r}^{t}+F_{l, h}^{t} \\
I^{t} \dot{\gamma}_{c}^{t} & =l_{f}^{t} F_{l, f}^{t} \cos \delta^{t}-l_{r}^{t} F_{l, r}^{t}-l_{h}^{t} F_{l, h}^{t}
\end{aligned}
$$

The lateral motion of the trailer is written as follows:

$$
m^{i}\left(\dot{w}_{c}^{i}+v_{c}^{i} \gamma_{c}^{i}\right)=F_{l, r}^{i}+F_{l, h}^{i}
$$

where $m^{i}, w_{c}^{i}, v_{c}^{i}, \gamma_{c}^{i}, F_{l, r}^{i}$ and $F_{l, h}^{i}$ represent the mass, the lateral and longitudinal velocities, the yaw rate of the CG of the trailer, the lateral forces on the rear wheel of the trailer and the hitch point, respectively. 
Table 1: NOMENCLATURE

\begin{tabular}{ll}
\hline Parameter & Description \\
\hline$m$ & Mass \\
$I$ & Moment of inertia around the vertical axis \\
$v$ & Longitudinal velocity \\
$w$ & Lateral velocity \\
$\psi$ & Yaw angle \\
$\gamma$ & Yaw rate \\
$\delta$ & Steering angle \\
$\beta$ & Angle between the center of gravity $(\mathrm{CG})$ of the tractor \\
$\lambda$ & and the CG of the drawbar \\
& Angle between the CG of the tractor \\
$l$ & and the CG of the trailer \\
$F$ & Distance \\
$C$ & Force \\
$\alpha$ & Cornering stiffness \\
$\sigma$ & Side-slip angle \\
\hline Superscript & Relaxation length \\
\hline $\mathrm{t}$ & Tractor \\
$\mathrm{i}$ & Trailer \\
\hline Subscript & \\
\hline $\mathrm{f}$ & front wheel \\
$\mathrm{r}$ & rear wheel \\
$\mathrm{c}$ & center of gravity \\
$\mathrm{h}$ & hitch point \\
$\mathrm{l}$ & lateral \\
$\mathrm{t}$ & traction \\
\hline & \\
\hline
\end{tabular}

Similarly, the dynamic equation for the yaw motion of the trailer can be written as:

$$
I^{i} \dot{\gamma}^{i}=m^{i} l_{h}^{i}\left(\dot{w}_{c}^{i}+v_{c}^{i} \gamma_{c}^{i}\right)-\left(l_{h}^{i}+l_{r}^{i}\right) F_{l, r}^{i}
$$

where $I^{i}, l_{h}^{i}$ and $l_{r}^{i}$ represent the moment of inertia of the trailer, the distances between the hitch point and the CG of the trailer and the rear axle and the CG of the trailer, respectively. Like the yaw inertial moment of the tractor, the yaw inertial moment of the trailer can be estimated as in (3).

The velocities of the CG of the trailer can be written with respect to the velocities of the CG of the tractor:

$$
\begin{aligned}
v_{c}^{i} & =v_{h}^{i}=v_{c}^{t} \cos \lambda-\left(w_{c}^{t}-l_{h}^{t} \gamma_{c}^{t}\right) \sin \lambda \\
w_{c}^{i} & =w_{h}^{i}-l_{h}^{i} \gamma_{c}^{i}=v_{c}^{t} \sin \lambda+\left(w_{c}^{t}-l_{h}^{t} \gamma_{c}^{t}\right) \cos \lambda-l_{h}^{i} \gamma_{c}^{i}
\end{aligned}
$$

where $\lambda$ is the total angle difference between the yaw angles of the tractor and trailer. In other words, it is equal to the summation of the angle $\beta$ between the tractor and drawbar and the steering angle $\delta^{i}$ of the trailer.

As the traction forces are neglected, the longitudinal acceleration $\dot{v}_{c}^{t}$ can be set to zero. As a results, the lateral acceleration of the trailer can be obtained by taking the time derivative of (9):

$$
\dot{w}_{c}^{i}=v_{c}^{t} \dot{\lambda} \cos \lambda+\left(\dot{w}_{c}^{t}-l_{h}^{t} \dot{\gamma}_{c}^{t}\right) \cos \lambda-\left(w_{c}^{t}-l_{h}^{t} \gamma_{c}^{t}\right) \dot{\lambda} \sin \lambda-l_{h}^{i} \dot{\gamma}^{i}
$$

where

$$
\dot{\lambda}=\gamma_{c}^{t}-\gamma_{c}^{i}
$$


From the lateral motion of the trailer in (6):

$$
F_{l, h}^{i}=m^{i}\left(\dot{w}_{c}^{i}+v_{c}^{i} \gamma_{c}^{i}\right)-F_{l, r}^{i}
$$

The relationship of the lateral force between the tractor and trailer at the hitch point is written considering the neglected traction forces as follows:

$$
F_{l, h}^{t}=-F_{l, h}^{i} \cos \lambda
$$

Equations (8), (10), (11) and (12) can be substituted in (13) to obtain the following equation:

$$
\begin{aligned}
F_{l, h}^{t}= & -m^{i} v_{c}^{t} \gamma_{c}^{t} \cos ^{2} \lambda-m^{i}\left(\dot{w}_{c}^{t}-l_{h}^{t} \dot{\gamma}_{c}^{t}\right) \cos ^{2} \lambda \\
& +m^{i}\left(w_{c}^{t}-l_{h}^{t} \gamma_{c}^{t}\right) \gamma_{c}^{t} \sin \lambda \cos \lambda+m^{i} l_{h}^{i} \dot{\gamma}_{c}^{i} \cos \lambda+F_{l, r}^{i} \cos \lambda
\end{aligned}
$$

By substituting (14) into (4), (5) and (7), the following equations are obtained for the vehicle dynamics.

$$
\begin{aligned}
& \left(m^{t}+m^{i} \cos ^{2} \lambda\right) \dot{w}_{c}^{t}-m^{i} l_{h}^{t} \cos ^{2} \lambda \dot{\gamma}_{c}^{t}-m^{i} l_{h}^{i} \cos \lambda \dot{\gamma}^{i}= \\
& -\left(m^{t}+m^{i} \cos ^{2} \lambda\right) v_{c}^{t} \gamma_{c}^{t}+m^{i} \sin \lambda \cos \lambda\left(w_{c}^{t}-l_{h}^{t} \gamma_{c}^{t}\right) \gamma_{c}^{t} \\
& +F_{l, f}^{t} \cos \delta+F_{l, r}^{t}+F_{l, r}^{i} \cos \lambda
\end{aligned}
$$

$$
\begin{aligned}
& \left(I^{t}+m^{i}\left(l_{h}^{t}\right)^{2} \cos ^{2} \lambda\right) \dot{\gamma}_{c}^{t}-m^{i} l_{h}^{t} \cos ^{2} \lambda \dot{w}_{c}^{t}+m^{i} l_{h}^{t} l_{h}^{i} \cos \lambda \dot{\gamma}^{i}= \\
& m^{i} l_{h}^{t} \cos ^{2} \lambda v_{c}^{t} \gamma_{c}^{t}-m^{i} l_{h}^{t} \sin \lambda \cos \lambda\left(w_{c}^{t}-l_{h}^{t} \gamma_{c}^{t}\right) \gamma_{c}^{t} \\
& F_{l, r}^{i} l_{h}^{t} \cos \lambda+l_{f}^{t} F_{l, f}^{t} \cos \delta-l_{r}^{t} F_{l, r}^{t}
\end{aligned}
$$

$$
\begin{aligned}
& \left(I^{i}+m^{i}\left(l_{h}^{i}\right)^{2}\right) \dot{\gamma}^{i}-m^{i} l_{h}^{i} \cos \lambda \dot{w}_{c}^{t}+m^{i} l_{h}^{t} l_{h}^{i} \cos \lambda \dot{\gamma}_{c}^{t}= \\
& m^{i} l_{h}^{i} \cos \lambda v_{c}^{t} \gamma_{c}^{t}-m^{i} l_{h}^{i} \sin \lambda\left(w_{c}^{t}-l_{h}^{t} \gamma_{c}^{t}\right) \gamma_{c}^{t}-\left(l_{h}^{i}+l_{r}^{i}\right) F_{l, r}^{i}
\end{aligned}
$$

\subsection{Lateral Tire Model}

The lateral tire forces are calculated in a linear model in which they are assumed to be proportional to the side-slip angles $[23,9,15]$ :

$$
F_{l, j}^{k}=-C_{\alpha, j}^{k} \alpha_{j}^{k} \quad j=\{f, r\}, \quad k=\{t, i\}
$$

where $C_{\alpha, j}^{k}$ and $\alpha_{j}^{k}, j=\{f, r\}, k=\{t, i\}$, represent the cornering stiffness of tires and the side-slip angles of the tractortrailer system, respectively. The tire cornering stiffness parameters correspond to the average slope of the lateral force characteristic. Although this method is not accurate for the larger side-slip angles [5], this model is usually used in online estimation cases due to its simplicity.

The tire side-slip angles must be calculated in order to determine the slip forces. The side-slip angles are written as follows:

$$
\begin{aligned}
\alpha_{f}^{t} & =\frac{w_{c}^{t}+l_{f}^{t} \gamma_{c}^{t}}{v_{c}^{t}}-\delta^{t} \\
\alpha_{r}^{t} & =\frac{w_{c}^{t}-l_{r}^{t} \gamma_{c}^{t}}{v_{c}^{t}} \\
\alpha_{r}^{i} & =\frac{w_{c}^{t}-l_{h}^{t} \gamma_{c}^{t}-\left(l_{h}^{i}+l_{r}^{i}\right) \gamma_{c}^{i}}{v_{c}^{t}}+\lambda
\end{aligned}
$$

As can be seen from the equations above, the side-slip angles cannot be calculated for the zero value of the longitudinal speed. As a solution to this problem, the relaxation length is defined as the amount of a tire rolls to reach the steady state side-slip angle. As can be seen from previous researches in agricultural vehicles, the relaxation length of a tire plays a very important role in steering motion $[6,21]$. Since a tire generates the steady state side-slip angle 
simultaneously, a first order mathematical model is used to describe the slip angle dynamics through the relaxation length. A first order differential equation for the side-slip angle can be written as follows:

$$
\dot{\alpha}=\frac{v_{c}^{t}}{\sigma}\left(\alpha_{0}-\alpha\right)
$$

where $\sigma$ is the relaxation length.

A relaxation length of 1.5 times the tire radius has been proposed for agricultural vehicles as it allows to obtain similar changes for a similar increase in velocity [4]. For passenger vehicles which have higher velocity than agricultural vehicles, a factor larger than 2 is typically selected [17].

By substituting (19) into (20), the three side-slip angles of the tires are written as follows:

$$
\begin{aligned}
\dot{\alpha}_{f}^{t} & =\frac{w_{c}^{t}+l_{f}^{t} \gamma_{c}^{t}-v_{c}^{t}\left(\delta^{t}+\alpha_{f}^{t}\right)}{\sigma_{f}^{t}} \\
\dot{\alpha}_{r}^{t} & =\frac{w_{c}^{t}-l_{r}^{t} \gamma_{c}^{t}-v_{c}^{t} \alpha_{r}^{t}}{\sigma_{r}^{t}} \\
\dot{\alpha}_{r}^{i} & =\frac{w_{c}^{t}-l_{h}^{t} \gamma_{c}^{t}-\left(l_{h}^{i}+l_{r}^{i}\right) \gamma^{i}+v_{c}^{t}\left(\lambda-\alpha_{r}^{i}\right)}{\sigma_{r}^{i}}
\end{aligned}
$$

where $\sigma_{f}^{t}, \sigma_{r}^{t}$ and $\sigma_{r}^{i}$ represent the relaxation length of the front and rear tires of the tractor, and the relaxation length of the tire of the trailer, respectively.

\subsection{Equations of Yaw Motion}

The angle, $\lambda$, at point $H$ can be defined in two ways:

- The total angle difference between the yaw angles of the tractor and trailer,

- The summation of the angle, $\beta$, between the tractor and drawbar, and the steering angle, $\delta^{i}$, of the trailer.

In this investigation, the former method is preferred to write the equations of motion of the tractor-trailer system. This results in having only one input for the system. Under these assumptions, the equations of motion of the tractortrailer system can be written as follows:

$$
M \dot{x}(t)=f(x(t))
$$

where

$$
M=\left[\begin{array}{ccccccc}
m^{t}+m^{i} \cos ^{2} \lambda & -m^{i} l_{h}^{t} \cos ^{2} \lambda & -m^{i} l_{h}^{i} \cos \lambda & 0 & 0 & 0 & 0 \\
-m^{i} l_{h}^{t} \cos ^{2} \lambda & I_{c}^{t}+m^{i}\left(l_{h}^{t}\right)^{2} \cos ^{2} \lambda & m^{i} l_{h}^{t} l_{h}^{i} \cos \lambda & 0 & 0 & 0 & 0 \\
-m^{i} l_{h}^{i} \cos \lambda & m^{i} l_{h}^{t} l_{h}^{i} \cos \lambda & I_{c}^{i}+m^{i}\left(l_{h}^{i}\right)^{2} & 0 & 0 & 0 & 0 \\
0 & 0 & 0 & 1 & 0 & 0 & 0 \\
0 & 0 & 0 & 0 & 1 & 0 & 0 \\
0 & 0 & 0 & 0 & 0 & 1 & 0 \\
0 & 0 & 0 & 0 & 0 & 0 & 1
\end{array}\right]
$$

$$
x(t)=\left[\begin{array}{lllllll}
w_{c}^{t} & \gamma_{c}^{t} & \gamma_{c}^{i} & \alpha_{f}^{t} & \alpha_{r}^{t} & \alpha_{r}^{i} & \lambda
\end{array}\right]^{T}
$$




$$
\begin{aligned}
& f_{1}=-m^{t} v_{c}^{t} \gamma_{c}^{t}-m^{i} \gamma_{c}^{t} \cos \lambda\left(v_{c}^{t} \cos \lambda-\left(w_{c}^{t}-l_{h}^{t} \gamma_{c}^{t} \sin \lambda\right)\right) \\
& -C_{\alpha, f}^{t} \alpha_{f}^{t} \cos \delta^{t}-C_{l, r}^{t} \alpha_{r}^{t}-C_{l, r}^{i} \alpha_{r f}^{i} \cos \lambda \\
& f_{2}=m^{i} l_{h}^{t} \gamma_{c}^{t} \cos \lambda\left(v_{c}^{t} \cos \lambda-\left(w_{c}^{t}-l_{h}^{t} \gamma_{c}^{t} \sin \lambda\right)\right) \\
& -l_{f}^{t} C_{l, f}^{t} \alpha_{f}^{t} \cos \delta^{t}+l_{r}^{t} C_{l, r}^{t} \alpha_{r}^{t}+l_{h}^{t} C_{l, r}^{i} \alpha_{r}^{i} \cos \lambda \\
& f_{3}=m^{i} l_{h}^{i} \gamma_{c}^{t}\left(v_{c}^{t} \cos \lambda-\left(w_{c}^{t}-l_{h}^{t} \gamma_{c}^{t} \sin \lambda\right)\right)+C_{l, r}^{i} \alpha_{r}^{i}\left(l_{h}^{i}+l_{r}^{i}\right) \\
& f_{4}=\frac{w_{c}^{t}+l_{f}^{t} \gamma_{c}^{t}-v_{c}^{t}\left(\delta^{t}+\alpha_{f}^{t}\right)}{\sigma_{f}^{t}} \\
& f_{5}=\frac{w_{c}^{t}-l_{r}^{t} \gamma_{c}^{t}-v_{c}^{t} \alpha_{r}^{t}}{\sigma_{r}^{t}} \\
& f_{6}=\frac{w_{c}^{t}-l_{h}^{t} \gamma_{c}^{t}-\left(l_{h}^{i}+l_{r}^{i}\right) \gamma^{i}+v_{c}^{t}\left(\lambda-\alpha_{r}^{i}\right)}{\sigma_{r}^{i}} \\
& f_{7}=\gamma_{c}^{t}-\gamma_{c}^{i} \\
& f(x(t))=\left[\begin{array}{lllllll}
f_{1} & f_{2} & f_{3} & f_{4} & f_{5} & f_{6} & f_{7}
\end{array}\right]^{T}
\end{aligned}
$$

\subsection{Parameter Identification}

As many of the model parameters cannot be measured directly, they have to be identified experimentally. In this investigation, the soil-tire interaction parameters described with cornering stiffnesses in Section 3 have to be estimated due to the fact that it is difficult to measure them in real-life. The masses $m^{t}$ and $m^{i}$, the inertia moments $I^{t}$ and $I^{i}$, the distances $l_{f}^{t}, l_{r}^{t}, l_{h}^{t}, l_{h}^{i}$ and $l_{r}^{i}$, the relaxation lengths $\sigma_{f}^{t}, \sigma_{r}^{t}$ and $\sigma_{r}^{i}$ are directly measurable. For the available real-time set up, these parameters are determined as $m^{t}=700 \mathrm{~kg}, m^{i}=100 \mathrm{~kg}, I^{t}=280 \mathrm{Nms}^{2}, I^{i}=42 \mathrm{Nms}^{2}, l_{f}^{t}=1.0 \mathrm{~m}$, $l_{r}^{t}=0.4 \mathrm{~m}, l_{h}^{t}=1.5 \mathrm{~m}, l_{h}^{i}=0.5 \mathrm{~m}$ and $l_{r}^{i}=0.8 \mathrm{~m}$. So, the parameters which have to be identified are the cornering stiffness for the front tire of the tractor $C_{\alpha, f}^{t}$, the cornering stiffness for the rear tire of the tractor $C_{\alpha, r}^{t}$ and the cornering stiffness for the tire of the trailer $C_{\alpha, r}^{i}$.

A parametric nonlinear least-squares identification is formulated as follows:

$$
\begin{array}{ll}
\min _{x(.), C_{\alpha, f}^{t}, C_{\alpha, r}^{t}, C_{\alpha, r}^{i}} & \sum_{k=1}^{n}\left(h\left(t_{k}\right)-h_{m}\left(t_{k}\right)\right)^{2} \\
\text { subject to } & \dot{x}(t)=M^{-1} f(x(t), u(t), p) \\
& \delta^{t}(t)=\delta_{m}^{t}\left(t_{k}\right) \\
& v^{t}(t)=v_{m}^{t}\left(t_{k}\right) \\
& 3000 \mathrm{~N} / \mathrm{rad} \leq C_{\alpha, f}^{t} \leq 25000 \mathrm{~N} / \mathrm{rad} \\
& 25000 \mathrm{~N} / \mathrm{rad} \leq C_{\alpha, r}^{t} \leq 150000 \mathrm{~N} / \mathrm{rad} \\
& 100 \mathrm{~N} / \mathrm{rad} \leq C_{\alpha, r}^{i} \leq 10000 \mathrm{~N} / \mathrm{rad} \quad \forall t \in[0, T]
\end{array}
$$

where $h_{m}\left(t_{k}\right)$ are the measurements, $h\left(t_{k}\right)$ is the output function of the system, $n$ is the number of measurements, $\delta_{m}^{t}$ and $v_{m}^{t}$ are the measured steering angle and speed of the tractor, respectively. The constraints given in (23) have been derived from values reported in literature [22,20]. Since a typical input to the steering mechanism of the tractor is a stepwise increase of the reference steering angle, the parameter identification results are obtained based-on step inputs for the steering angle of the tractor. During the identification process, the measurements and the output function of the system were the yaw rates of the tractor and trailer. The parameter identification procedure is performed in the 
$A C A D O$ code generation tool which is an open source software package for optimization problems $[11,12]$ and can handle constrained nonlinear optimization problems.

The identified parameters are found as $C_{\alpha, f}^{t}=14250 \mathrm{~N} / \mathrm{rad}, C_{\alpha, r}^{t}=65720 \mathrm{~N} / \mathrm{rad}$ and $C_{\alpha, r}^{i}=1481 \mathrm{~N} / \mathrm{rad}$. The measured yaw rates of the tractor and trailer, and the responses of the yaw dynamics model with the identified parameters are shown in Fig. 6. These figures show that the simulation results fit the measurements with a reasonable accuracy.

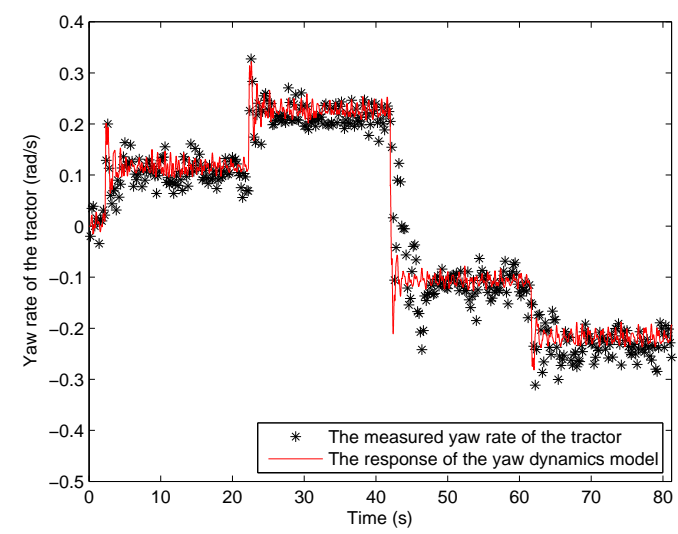

(a)

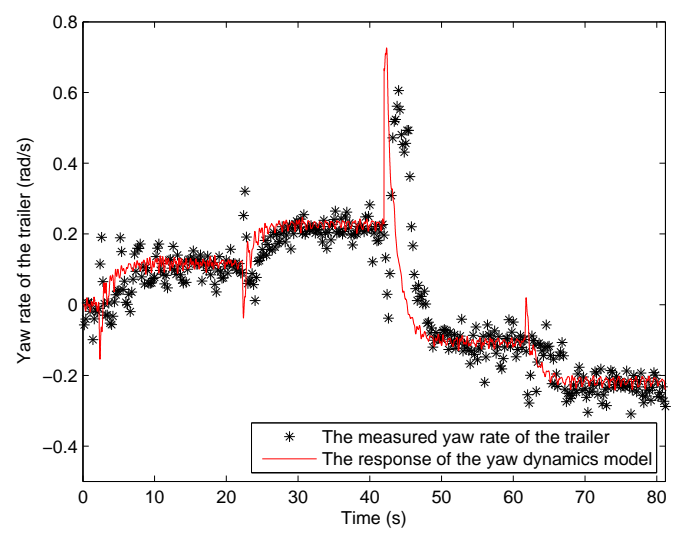

(b)

Figure 6: The parameter estimation results of the yaw dynamics model: (a) tractor (b) trailer

\section{The Longitudinal Dynamics Model}

First, a static model is derived to define the relationship between the hydrostat position, the engine speed and the longitudinal speed of the system. After the derivation of the static model, a dynamic model is proposed to define the relationship between the output of the static model and the actual longitudinal speed of the system. The structure of the longitudinal speed model is shown in Fig. 7 in which the grey squares represent linear models, while the black circle represents a nonlinear model. As can be seen from Fig. 7, it comprises a static nonlinearity sandwiched between linear blocks. This structure is known as a Wiener-Hammerstein structure [29].

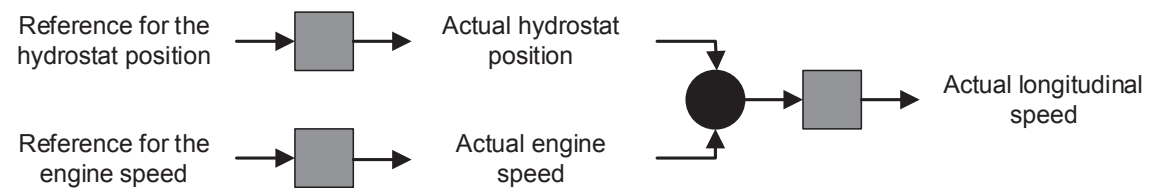

Figure 7: The overview of the longitudinal speed model.

\subsection{Static Model}

The static model is a 2D surface linking the hydrostat position and the diesel engine speed to the longitudinal speed of the tractor. First, a ramp signal has been applied to the hydrostat position at different engine speeds. The relation between the hydrostat position and the longitudinal speed for different engine speeds is shown in Fig. 8. As can be seen from Fig. 8, the relation is linear with a dead-zone in the range from $0 \%$ to $15 \%$. On the other hand, the relation between the diesel engine speed and the longitudinal speed is not completely linear. That is why a second-order 
polynomial function has been chosen for the engine speed while a first-order polynomial function has been chosen for the hydrostat position. The formulation between the hydrostat position, the engine speed and the longitudinal speed can be written as follows:

$$
v(H P, R P M)=p_{00}+p_{10} \times H P+p_{01} \times R P M+p_{11} \times H P \times R P M+p_{02} \times R P M^{2}
$$

where $v, H P$ and $R P M$ are the longitudinal speed, the hydrostat position and the engine speed, respectively. As can be seen from the formulation, the static model has one output and two inputs. After the parameter estimation process, the coefficients were found as $p_{00}=0.1467, p_{10}=2.44 \times 10^{-4}, p_{01}=-2.474 \times 10^{-4}, p_{11}=7.675 \times 10^{-6}$ and $p_{02}=2.908 \times 10^{-8}$.

The correspondence between the longitudinal speed simulated with the estimated model in (24) and the measured longitudinal speeds is illustrated in Fig. 8. It can be seen that a satisfactory accuracy has been achieved with the estimated parameters.

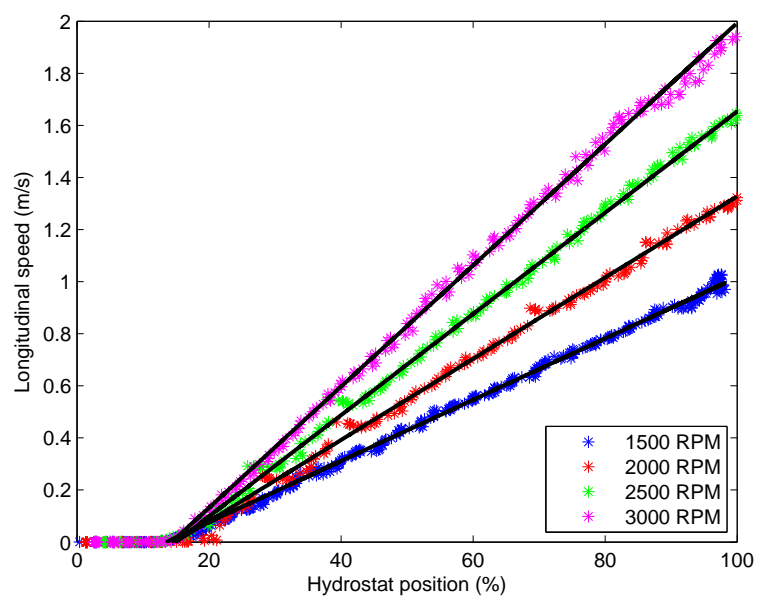

Figure 8: The measured relation between the hydrostat position and longitudinal speed.

\subsection{Dynamic Model}

In the previous subsection, it was assumed that there were no dynamic effects on the longitudinal speed model. However, this is not completely correct for real-time systems. Dynamic effects can be observed in the following three cases:

- between the reference and the actual hydrostat position,

- between the reference and the actual engine speed,

- between the output of the static model and the actual longitudinal speed of the system.

The dynamic behavior indicated in the first two items can be checked by using an odd multisine as an excitation signal $[28,27]$. The excitation signals, which have a frequency content up to $10 \mathrm{~Hz}$, were fed to the subsystems as references and the outputs of the subsystems (the actual hydrostat position and the actual engine speed) were observed. It is seen that the linear contributions are dominant until $6 \mathrm{~Hz}$ with respect to the results of the odd multisine signals. Therefore, a multisine signal, which must have a frequency content up to $6 \mathrm{~Hz}$, is needed to excite all dynamics effects of the subsystems. This is not a convenient method for this application due to the fact that a controller cannot apply a signal at high frequencies for the sake of the operator comfort. Since a controller will not excite these dynamics, they can be neglected. This results in having only one dynamic effect which is between the output of the static model and the actual longitudinal speed of the system. 
Since the typical input for a longitudinal speed controller will be a stepwise increase of the reference longitudinal speed, the dynamics have been identified based-on step inputs for the hydrostat position at different engine speed values. As can be seen from Fig. 9, the response of the longitudinal speed is a similar one to a first order system. The following first-order transfer function is proposed for the dynamic model:

$$
G(s)=\frac{K}{\tau s+1}
$$

The following parameters were estimated as $K=1.0327$ and $\tau=2.0585$ by using the step responses of the longitudinal speed at $2500 \mathrm{rpm}$. Since the static model has been derived for every diesel engine speed measurement, these parameters for the dynamic model are also valid for different diesel engine speed values. The corresponding fit of the simulated longitudinal speed profile to the measured longitudinal speed profile is illustrated in Fig. 9. It is to be noted that since the longitudinal speed is measured by encoders mounted on the rear wheels of the tractor, the longitudinal speed $v_{c}^{t}$ in Section 3 is equal to the measured wheel speed multiplied by the forward slip ratio.

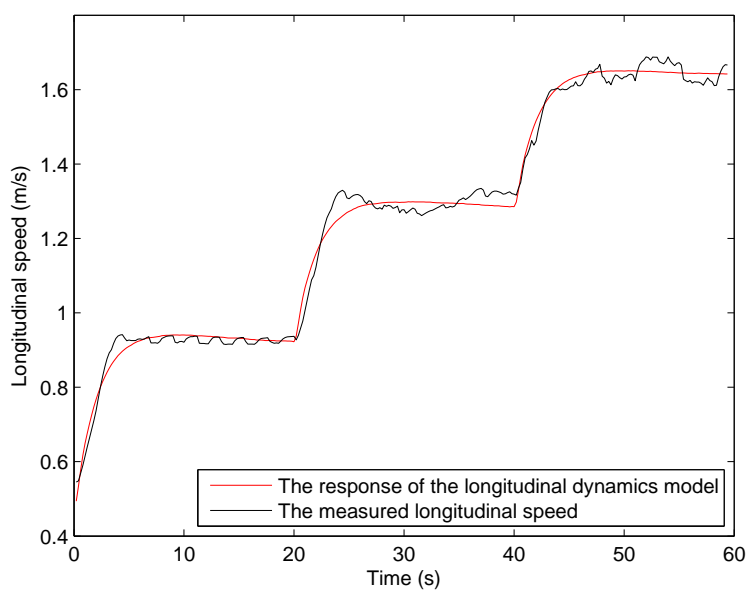

Figure 9: The response of the longitudinal dynamics model and the measured longitudinal speed.

\section{Identification of the Steering Mechanisms of the Tractor and Trailer}

When the excitation signal was applied to the steering mechanisms in an open-loop fashion, it was observed that the front wheels of the tractor and the actuator of the trailer reached their limits. As a solution to this drifting problem, the systems were controlled with a P controller for each subsystem, and then the closed-loop systems were identified. The schematic diagram of the identification processes is shown in Fig. 10.

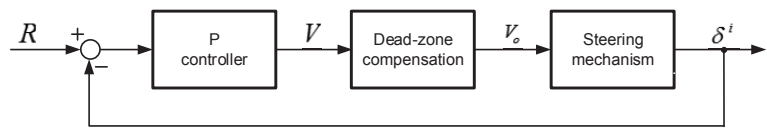

Figure 10: The schematic diagram of the identification processes for the steering mechanisms.

The oil flow to the steering actuators connected to the tractor and trailer is controlled with electro-hydraulic valves which are characterized by some static nonlinearities: dead-band region and saturation. Thus, several data sets were 
collected to estimate the steady state characteristics of the steering valves. The model from volt-to-volt in (26) has been identified to invert the static nonlinearities of the steering valves. Once the steady state characteristic of the steering valves is known, it is assumed that the valve nonlinearity can be perfectly inverted. The used dead-zone-compensation (DZC) is written as follows:

$$
\mathrm{DZC}= \begin{cases}\text { if } V<0 & V_{o}=V+6-\varepsilon \\ \text { if } V>0 & V_{o}=V+6+\varepsilon \\ \text { if } V=0 & V_{o}=6\end{cases}
$$

where $V$ and $V_{o}$ are respectively the input and the output of the DZC, $\varepsilon$ is a numerical value equal to 0.9 and 0.5 for the tractor and trailer, respectively.

\subsection{The Model of the Steering Mechanism of the Tractor}

Since the dynamic behaviour of the steering mechanism of the tractor is not changed with respect to the engine speed, the effect of the engine speed will not be an issue in this subsection. This results in a dynamic model consisting of only one input, the voltage to the actuator, and only one output, the steering angle of the front wheels.

The steering mechanism of the tractor with the proposed DZC is excited with an odd-odd multisine to detect the level of nonlinear distortions [28, 27]. In this technique, only a well chosen set of frequency lines with a periodic excitation is excited. The rest of the frequency lines are not excited intentionally. It is observed that the level of the nonlinearities is similar to the level of the noise, and the nonlinear contributions are about $25 d B$ smaller than the linear contributions in the excited frequency band. This suggests that the steering mechanism can be described with a linear model.

The steering mechanism of the tractor is a high order model. However, since the natural frequency of the valve is higher than the natural frequency of the steering system, it is possible to simplify the model of the steering mechanism as a first order model for the rate of the steering angle and second order system model for the steering angle [18, 26]. As a result, the steering mechanism of the tractor can be considered as a mass-damper system for the steering angle. The relation between the voltage to the valve and the steering angle can be modeled as follows:

$$
\frac{\delta^{t}(s)}{V(s)}=\frac{K}{s(\tau s+1)}
$$

The transfer function for the closed-loop system can be written as follows:

$$
\frac{\delta^{t}(s)}{R(s)}=\frac{P K}{s(\tau s+1)+P K}
$$

where the $\mathrm{P}$ controller coefficient is set to 5 during the experiments.

A multisine signal with a frequency content $0.015-1.5 \mathrm{~Hz}$ has been applied to the steering mechanism as an excitation signal. The parameters of the model have been identified by using NLS frequency domain identification approaches based on FRF measurements. In Fig. 11, the measured FRF and the FRF of the identified model in the closed-loop fashion are shown. It has been observed during the experiments that it is inconvenient to give an excitation signal to the steering system larger than $1.5 \mathrm{~Hz}$. The reason is due to the lack of input signal above $1.5 \mathrm{~Hz}$, and the frequency domain response of the system is quite noisy to be analyzed. However, the range until $1.5 \mathrm{~Hz}$ is enough to capture the second order peak at $0.8 \mathrm{~Hz}$.

By nonlinear least squares fitting of the second order model to the estimated empirical transfer function, as illustrated in Fig. 11, the following model has been estimated for the steering system:

$$
\frac{\delta^{t}(s)}{R(s)}=\frac{43}{s^{2}+7.7 s+45}
$$

As can be seen from (29), $P K$ values in the numerator and in the denominator are not the same due to the noise in the measurements, calculation errors and perturbation effect. $P K$ value in the denominator is used to determine the transfer function of the steering mechanism. By using (29), the transfer function of the steering mechanism with the DZC is obtained as follows:

$$
\frac{\delta^{t}(s)}{V(s)}=\frac{1.17}{s(0.13 s+1)}
$$



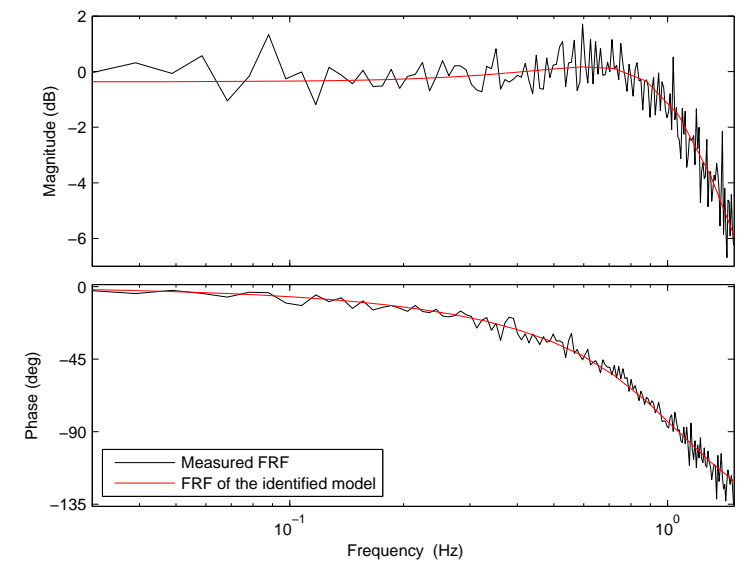

Figure 11: Measured FRF and FRF of the identified model for the steering mechanism of the tractor

\subsection{The Model of Steering Mechanism of the Trailer}

Similar to the speed model in Section 4, a static model has been derived to define the relationship between the voltage to the electro-hydraulic valve, the engine speed and the steering rate of the trailer. After the derivation of the static model, a dynamic model is proposed to define the relationship between the output of the static model and the actual steering angle of the trailer. The model structure of the steering angle of the trailer is shown in Fig. 12 in which the grey squares represent linear models, whereas the black circle represents a nonlinear model.

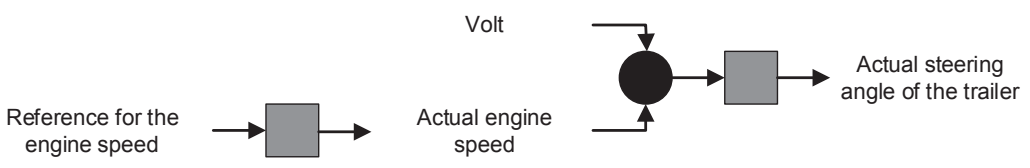

Figure 12: The overview of the steering angle model of the trailer.

\subsubsection{Static Model}

The static model is a 2D surface linking the voltage and the engine speed to the steering rate of the trailer. Firstly, step inputs are fed to the steering valve as voltage at different engine speeds in order to obtain the relation between voltage and steering rate for different engine speeds as shown in Fig. 13. As can be seen from Fig. 13, the slopes of the curves and the saturations for the relation between the voltage and the steering rate of the trailer are not the same for positive and negative values of the voltage. Due to this asymmetric behaviour of the steering mechanism of the trailer, two different models are proposed to describe the static characteristic of the system as follows:

$$
\begin{array}{ll}
\text { if } V<0 & \dot{\delta}^{i}=-0.1107 \times V+0.5465 \times 10^{-1} \\
& \dot{\delta}_{\text {max }}^{i}=0.135 \times 10^{-3} \times R P M-0.1325 \\
\text { if } V>0 & \dot{\delta}^{i}=-0.1582 \times V-0.4776 \times 10^{-1} \\
& \dot{\delta}_{\text {min }}^{i}=-0.175 \times 10^{-3} \times R P M+0.1825 \\
\text { if } V=0 & \dot{\delta}^{i}=0
\end{array}
$$

where $\dot{\delta}^{i}, V$ and $R P M$ are the steering rate of the trailer, the voltage and the engine speed, respectively. As can be seen from the formulation, the static models have one output and two inputs. The slopes of the static models are similar for 
every engine speed, while the values of the saturations are different from each other. The parameter estimation results for the static model are shown in Fig. 13. It can be seen from Fig. 13 that the static model in (31) gives satisfactory estimation results.

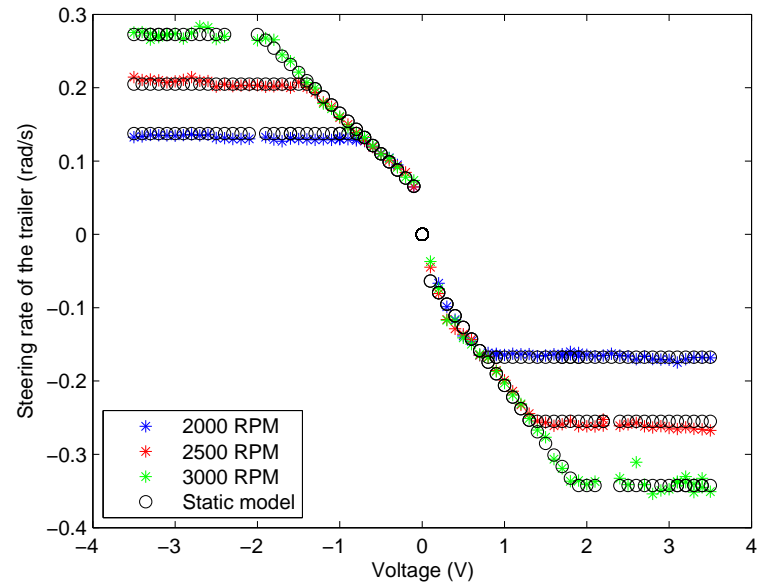

Figure 13: The measured relation between the voltage and the steering rate of the trailer.

\subsubsection{Dynamic Model}

In this subsection, the effect of the dynamics between the output of the static model and the actual steering angle of the trailer is identified. The steering mechanism of the trailer with the proposed DZC is excited with an odd-odd multisine to detect the level of nonlinear distortions [28, 27]. The frequency spectrum of the response of the steering mechanism of the trailer to a random odd-odd multisine excitation is shown in Fig. 14. It can be seen that the contribution of the nonlinearities to the total response is as large as the linear contribution after $0.25 \mathrm{~Hz}$. Since the nonlinear contributions are dominant after $0.25 \mathrm{~Hz}$, a linear model can be derived until $0.25 \mathrm{~Hz}$.

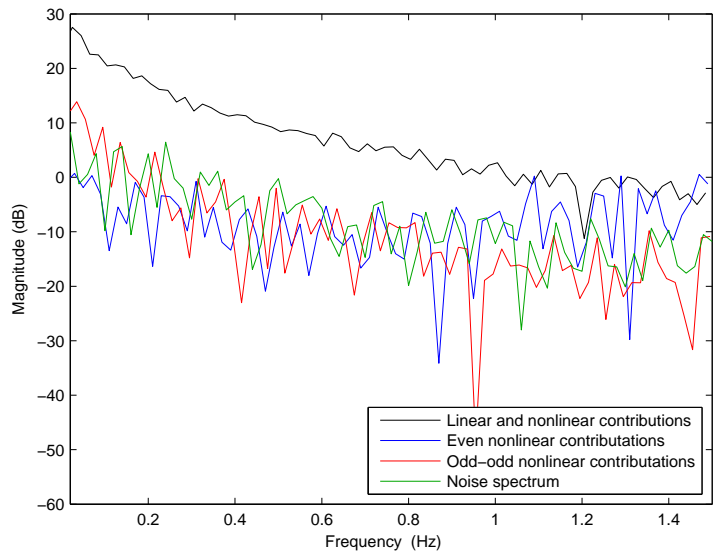

Figure 14: The analysis of nonlinear contributions for the steering mechanism of the trailer 
Based-on the considerations above, a multisine signal with a frequency range $0.0015-0.25 \mathrm{~Hz}$ has been applied to the steering mechanism of the trailer as an excitation signal. The model parameters are identified by using a nonlinear least squares frequency domain identification approach based on FRF measurements.

The difference between the output of the static model and the actual steering rate of the trailer is considered as a second-order system. Since the steering angle of the trailer is supposed to be the output of the transfer function, a free integrator is included in the denominator. The relation between the output of the static model and the actual steering angle of the trailer can be modeled as follows:

$$
\frac{\delta^{i}(s)}{V(s)}=\frac{K}{s\left(s^{2}+2 \zeta \omega+\omega^{2}\right)}
$$

where $K, \zeta$ and $\omega$ are the gain, damping ratio and the angular velocity of the transfer function. These parameters were estimated as $K=-3.39, \zeta=0.70$ and $\omega=2.26$. The parameter estimation results for the dynamic model are shown in Fig. 15. It can be seen from Fig. 15 that a satisfactory identification accuracy has been achieved.

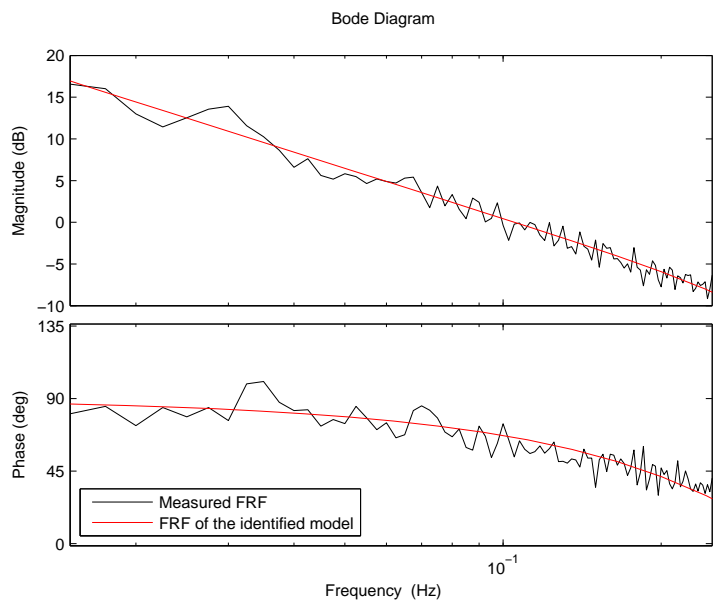

Figure 15: Measured FRF and FRF of the identified model for the steering mechanism of the trailer

\section{Conclusions}

First, a nonlinear model for the yaw dynamics of a tractor-trailer system has been derived and validated in this study. Second, the longitudinal dynamics and the steering mechanism of the trailer have been modelled and identified based on the static and dynamic models by taking different diesel engine speeds into account. While the models have been formulated based on the physical insights coming from the system, their parameters have been determined based on the experimental data. Eventually, a complete model for all the subsystems in a tractor-trailer system considering different engine speed measurements has been achieved. Benefitting from its simplicity, number of papers prefer to use kinematic models in their controller design. However, for a better understanding of the dynamic behaviour of the systems to be controlled, a dynamic model is needed. Since the proposed model is dynamic and nonlinear as well as it contains some uncertain parameters, it is believed that it has a great potential to be a benchmark for the performance evaluation of several model-based control algorithms. Regarding the models obtained, the nonlinearities are more visible on subsystems where the diesel engine speed has a big influence. For instance, the steering mechanism of the trailer is more nonlinear when compared to its tractor counterpart. A similar conclusion is also valid for the longitudinal speed model too. 


\section{Acknowledgement}

This work has been carried out within the framework of the project IWT-SBO 80032 (LeCoPro) of the Institute for the Promotion of Innovation through Science and Technology in Flanders (IWT-Vlaanderen). We would like to thank Mr. Soner Akpinar for his technical support for the preparation of the experimental set up.

\section{References}

[1] Backman, J., Oksanen, T., Visala, A., 2010. Nonlinear model predictive trajectory control in tractor-trailer system for parallel guidance in agricultural field operations, in: Proceedings of the Agricontrol 2010, IFAC International Conference. Agricontrol 2010, Kyoto, Japan, December 6-8, 2010.

[2] Backman, J., Oksanen, T., Visala, A., 2012. Navigation system for agricultural machines: Nonlinear model predictive path tracking. Computers and Electronics in Agriculture 82, 32 - 43.

[3] Bell, T., 2000. Automatic tractor guidance using carrier-phase differential \{GPS \}. Computers and Electronics in Agriculture 25, 53 - 66.

[4] Bevly, D., Gerdes, J., Bradford, W., 2002. A new yaw dynamic model for improved high speed control of a farm tractor. Journal of Dynamic Systems, Measurement, and Control 124, 659 - 667.

[5] Clover, C.L., Bernard, J.E., 1998. Longitudinal tire dynamics. Vehicle System Dynamics 29, $231-259$.

[6] Crolla, D.A., 1983. The steering behavior of off-road vehicles, in: Proceedings of 8th IAVSD-Symp., Cambridge, MA.

[7] Feng, L., He, Y., Bao, Y., Fang, H., 2005. Development of trajectory model for a tractor-implement system for automated navigation applications, in: Proceedings of the IEEE Instrumentation and Measurement Technology Conference 2005, pp. 1330-1334.

[8] Garrott, W.R., Monk, M.W., Chrstos, J.P., 1998. Vehicle inertial parametersmeasured values and approximations, in: SAE Passenger Car Meeting and Exposition, Detroit, MI.

[9] Geng, C., Mostefai, L., Dena, M., Hori, Y., 2009. Direct yaw-moment control of an in-wheel-motored electric vehicle based on body slip angle fuzzy observer. IEEE Transactions on Industrial Electronics 56, $1411-1419$.

[10] Hiremath, S.A., van der Heijden, G.W., van Evert, F.K., Stein, A., ter Braak, C.J., 2014. Laser range finder model for autonomous navigation of a robot in a maize field using a particle filter. Computers and Electronics in Agriculture 100, $41-50$.

[11] Houska, B., Ferreau, H.J., Diehl, M., 2011a. Acado toolkitan open-source framework for automatic control and dynamic optimization. Optimal Control Applications and Methods 32, 298 - 312.

[12] Houska, B., Ferreau, H.J., Diehl, M., 2011b. An auto-generated real-time iteration algorithm for nonlinear MPC in the microsecond range. Automatica 47, $2279-2285$.

[13] Julian, A., 1971. Design and performance of a steering control system for agricultural tractors. Journal of Agricultural Engineering Research $16,324-336$.

[14] Karkee, M., Steward, B.L., 2010. Study of the open and closed loop characteristics of a tractor and a single axle towed implement system. Journal of Terramechanics 47, $379-393$

[15] Kayacan, E., Kayacan, E., Ramon, H., Saeys, W., 2013. Modeling and identification of the yaw dynamics of an autonomous tractor, in: Control Conference (ASCC), 2013 9th Asian, pp. 1-6.

[16] Kraus, T., Ferreau, J., Kayacan, E., Ramon, H., Baerdemaeker, J.D., Diehl, M., Saeys, W., 2013. Moving horizon estimation and nonlinear model predictive control for autonomous agricultural vehicles. Computers and Electronics in Agriculture 98, 25 - 33.

[17] Loeb, J., Guenther, D., Chen, H., 1990. Lateral stiffness, cornering stiffness, and relaxation length of the pneumatic tire, in: SAE International Congress and Exposition, Detroit, MI.

[18] Mas, F., Zhang, Q., Hansen, A., 2011. Mechatronics and Intelligent Systems for Off-Road Vehicles. Springer.

[19] Matveev, A.S., Hoy, M., Katupitiya, J., Savkin, A.V., 2013. Nonlinear sliding mode control of an unmanned agricultural tractor in the presence of sliding and control saturation. Robotics and Autonomous Systems 61, 973 - 987.

[20] Metz, L.D., 1993. Dynamics of four-wheel-steer off-highway vehicles. SAE Paper No. 930765.

[21] Owen, R.H., Bernard, J.E., 1982. Directional dynamics of a tractor-loader-backhoe. Vehicle System Dynamics 11, 251 - 265.

[22] Pearson, P., Bevly, D.M., 2007. Modeling and validation of hitch loading effects on tractor yaw dynamics. Journal of Terramechanics 44, $439-450$.

[23] Piyabongkarn, D., Rajamani, R., Grogg, J.A., Lew, J.Y., 2009. Development and experimental evaluation of a slip angle estimator for vehicle stability control. IEEE Transactions on Control Systems Technologhy 17, $78-88$.

[24] Reid, J., Searcy, S., 1987. Vision-based guidance of an agriculture tractor. Control Systems Magazine, IEEE 7, 39-43.

[25] Rekow, A., of Aeronautics, S.U.D., Astronautics, 2001. System Identification, Adaptive Control and Formation Driving of Farm Tractors. Stanford University.

[26] Saeys, W., Wallays, C., Engelen, K., Ramon, H., Anthonis, J., 2008. An automatic depth control system for shallow slurry injection, part 2: Control design and field validation. Biosystems Engineering 99, $161-170$.

[27] Schoukens, J., Pintelon, R., Dobrowiecki, T., Rolain, Y., 2005. Identification of linear systems with nonlinear distortions. Automatica 41, $491-504$.

[28] Vanhoenacker, K., Schoukens, J., 2003. Detection of nonlinear distortions with multisine excitations in the case of nonideal behavior of the input signal. IEEE Transactions on Instrumentation and Measurement 52, $748-753$.

[29] Wong, H.K., Schoukens, J., Godfrey, K., 2012. Analysis of best linear approximation of a wiener-hammerstein system for arbitrary amplitude distributions. Instrumentation and Measurement, IEEE Transactions on 61, 645-654. 